\title{
Routine screening in the general hospital: what happens after discharge to those identified as at risk of dementia?
}

\author{
Authors: Ana Phelps, ${ }^{A}$ Bethany Kingston, ${ }^{B}$ Rose M Wharton ${ }^{C}$ and Sarah T Pendlebury ${ }^{\mathrm{D}}$
}

\begin{abstract}
Cognitive screening is recommended for older patients with unplanned hospital admission. We determined rates of reassessment/specialist memory referral after routine inclusion of at risk of dementia status in discharge documentation to primary care. Questionnaires were sent to relevant GP practices on consecutive patients aged $\geq 75$ years identified as at risk and discharged 6 months earlier. Among 53 patients (mean age $\pm S D=87.3 \pm 6.0$ years, mean $\pm S D$ Abbreviated Mental Test Score $=4.4 \pm 2.7), 49(92 \%)$ patients had been reviewed since discharge, and 12/43 (28\%) without previously known cognitive problem had had a cognitive reassessment. The most common reasons for non-assessment/referral included clinical factors (eg terminal illness/comorbidities) $(n=15)$ and patient/ family wishes $(n=5)$ and that confusion was expected in unwell older patients $(n=5)$. Routine cognitive reassessment/specialist referral appears unjustified in patients identified as at risk of dementia during unplanned hospital admission. However, the prognostic value of delirium/confusion in acute illness is under-recognised and could be used to highlight those at risk.
\end{abstract}

KEYWORDS: at-risk, dementia, hospitalisation, primary care, prognosis

\section{Introduction}

Over $50 \%$ of older ( $\geq 75$ years) patients on acute general medicine wards have cognitive impairment; however, the majority do not have a pre-admission diagnosis ${ }^{1-3}$ because either an existing cognitive impairment/dementia has not been diagnosed or recognised ${ }^{1,4}$ or acute illness and hospitalisation has resulted in a sudden cognitive deterioration. Delirium occurs in over one third of those aged $\geq 75$ years $^{5,6}$ and transient cognitive impairment without delirium is common even with less serious illness. ${ }^{7}$

Recognition of the high prevalence of previously undiagnosed dementia in older hospitalised patients resulted in the

Authors: ${ }^{\text {A }}$ consultant orthogeriatrician, Stoke Madeville Hospital,

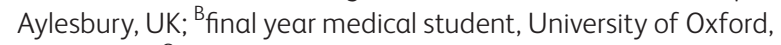

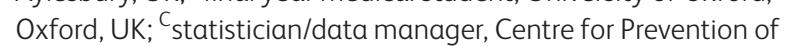
Stroke and Dementia, University of Oxford, Oxford, UK; ${ }^{\mathrm{D}}$ consultant physician and associate professor, Oxford University Hospitals NHS Trust and University of Oxford, Oxford, UK introduction of the dementia CQUIN (Commissioning for Quality and Innovation) in 2012, requiring routine screening of older inpatients with unplanned admission to identify those at risk of dementia using a single 'memory question' followed by further assessment in those with a memory complaint. ${ }^{8}$ The introduction of the dementia screening requirement generated some controversy with concerns around the lack of evidence base and the reliability of the single question. 3,9-11 Although there is no longer a financial incentive, individual hospital dementia screening rates are still the subject of national scrutiny. ${ }^{12}$

In our institution, we have developed a routine on-admission cognitive screening process for older patients that fulfils the original dementia CQUIN screening requirements while focusing on identifying objective cognitive deficits, including delirium $^{13}$ not reliably identified by the single memory question. ${ }^{3}$ Those identified as at risk of dementia are flagged to GPs via discharge documentation and are not routinely referred to specialist services during admission because cognitive impairment is associated with high mortality and morbidity ${ }^{6,14-16}$ and may not be the focus of the admission encounter. GPs may also be best placed to decide together with patients and families whether onward referral for specialist memory assessment is desirable.

We therefore performed a quantitative and qualitative study to determine the actions taken in primary care as a result of discharge documentation of at risk of dementia status. We determined rates of cognitive reassessment in the community and specialist referral, and reasons for non-reassessment/ referral. We intended to use the findings to improve the care of patients with cognitive impairment across the primary/ secondary care interface.

\section{Methods}

All patients aged $\geq 75$ years with unplanned admission to the Oxford University Hospitals NHS Foundation Trust have an on-admission validated cognitive screen, ${ }^{3,6,17}$ which includes the Abbreviated Mental Test Score (AMTS) ${ }^{18}$ and Confusion Assessment Method ${ }^{19}$ for delirium, and recording of known prior diagnosis of dementia. At risk of dementia status is defined as AMTS $\leq 8$ according to published cut-offs ${ }^{3}$ or delirium in those without a known prior diagnosis of dementia. 
1. On receipt of the notification letter, were any of the following done? Please tick all that apply:

The low AMTS was coded

Which Read code was used?

The letter was scanned and incorporated in the record

The AMTS score was noted in the record

Other

Please specify

2. Have you/a colleague seen the patient since the above admission?

YES

NO

3. If yes, was this in response to the notification letter?

YES

NO

4. Has their cognition been reassessed? (ie GPCog, AMTS, MOCA, MMSE etc)

$\begin{array}{lll}\text { YES } & \square \quad \text { Please state test used and result } \\ \text { NO } \quad \square \quad \text { Please state why not }\end{array}$

5. Has the patient been referred to a memory clinic, CMHT or geratology clinic for further assessment?

YES

NO

6. Has the patient been diagnosed with dementia?
YES
If so, by whom

NO

7. Has the patient received another (non-dementia) cognitive diagnosis, eg mild cognitive impairment?

YES $\square \quad$ If so, by whom

NO

8. Please add any comments on the process below:

Fig 1. Questionnaire sent to GPs. AMTS = Abbreviated Mental Test Score; CMHT = community mental health team; GPCog = GP cognitive screen; MMSE = mini-mental state examination; $\mathrm{MOCA}=$ Montreal Cognitive Assessment. 
At the time of this study, data on at-risk status for those who stayed in hospital $\geq 72$ hours was collected in line with national requirements ${ }^{8}$ and patient at-risk status was communicated to GPs on discharge via a notification letter containing the cognitive screening results sent separately from the patient's other discharge documentation.

The current study used a consecutive sample of 199 patients discharged at least 6 months earlier in whom at risk of dementia status had been identified during hospitalisation. Questionnaires (Fig 1), developed in collaboration with the clinical commissioning group's primary care leads in dementia, were sent to relevant primary care practices to determine the actions taken in primary care regarding the patient's at risk of dementia status. Data from returned questionnaires were entered into an anonymised database (by AP and BK). GP comments were extracted verbatim from the free comments box on the questionnaire and grouped by theme. The study was undertaken to facilitate the optimal management of patients with comorbid cognitive disorders, was approved by the divisional management and registered with the Oxford University Hospitals Audit Team, audit registration (datix) number 2981.

\section{Results}

A flow diagram of the questionnaire data is shown in Fig 2. Questionnaires were returned for 67/199 patients (34\%).

However, 14 questionnaires were blank ( $\mathrm{n}=4$ patient deceased, $n=6$ notification letter not found/no time, $n=3$ blank with no reason given, $\mathrm{n}=1$ patient transferred out). There were no differences in age or admission AMTS scores between those with and without returned questionnaires (mean \pm SD age $=86.8 \pm 6.2$ years, median age $=87$ (IQR 8392) years, range $75-99$ years versus $86.3 \pm 6.1$ years, median age $=87($ IQR $82-91)$ years, range $75-102$ years; mean \pm SD AMTS $=5.2 \pm 2.9$ versus $4.6 \pm 2.9$ ). The notification letter had been scanned and incorporated into the patient's documents file by primary care staff for 41/53 completed questionnaires $(77 \%$; mean \pm SD age $=87.3 \pm 6.0$ years, mean \pm SD AMTS $=$ $4.4 \pm 2.7$ ), but the low AMTS was only coded (READ code 388L) in six patients. In five patients, the AMTS score was not found. Of the 53 patients with completed questionnaires, $49(92 \%)$ had been seen in primary care since discharge from hospital.

In 10/53 patients, notification of at risk of dementia status letters had been sent inappropriately because dementia or another memory problem had been known to primary care but not to the hospital team: five patients (10\%) had mild cognitive impairment or another memory problem and five (10\%) had dementia although in one case, the diagnosis had been made in the memory clinic just prior to admission. In the remaining 43 without a previously identified cognitive problem, 12 (28\%) had cognition reassessed ( $\mathrm{n}=3 \mathrm{GP}$ assessment of cognition, $\mathrm{n}=3$ six-item cognitive impairment test, $\mathrm{n}=3$ mini-mentalstate-examination, $n=3$ 'informal' assessment/not specified), of whom five (12\%) were referred for specialist assessment. Of those who were referred for specialist assessment, four received a new dementia diagnosis $(\mathrm{n}=1$ memory clinic, $\mathrm{n}=1$ community mental health team, $\mathrm{n}=1$ GP specialist clinic, $\mathrm{n}=1$ geriatrician/physician).
Questionnaires sent to GPs, $n=199$ Consecutive patients $\geq 75$ years in whom notification letters had been sent on discharge to GPs concerning the patient's at risk of dementia status

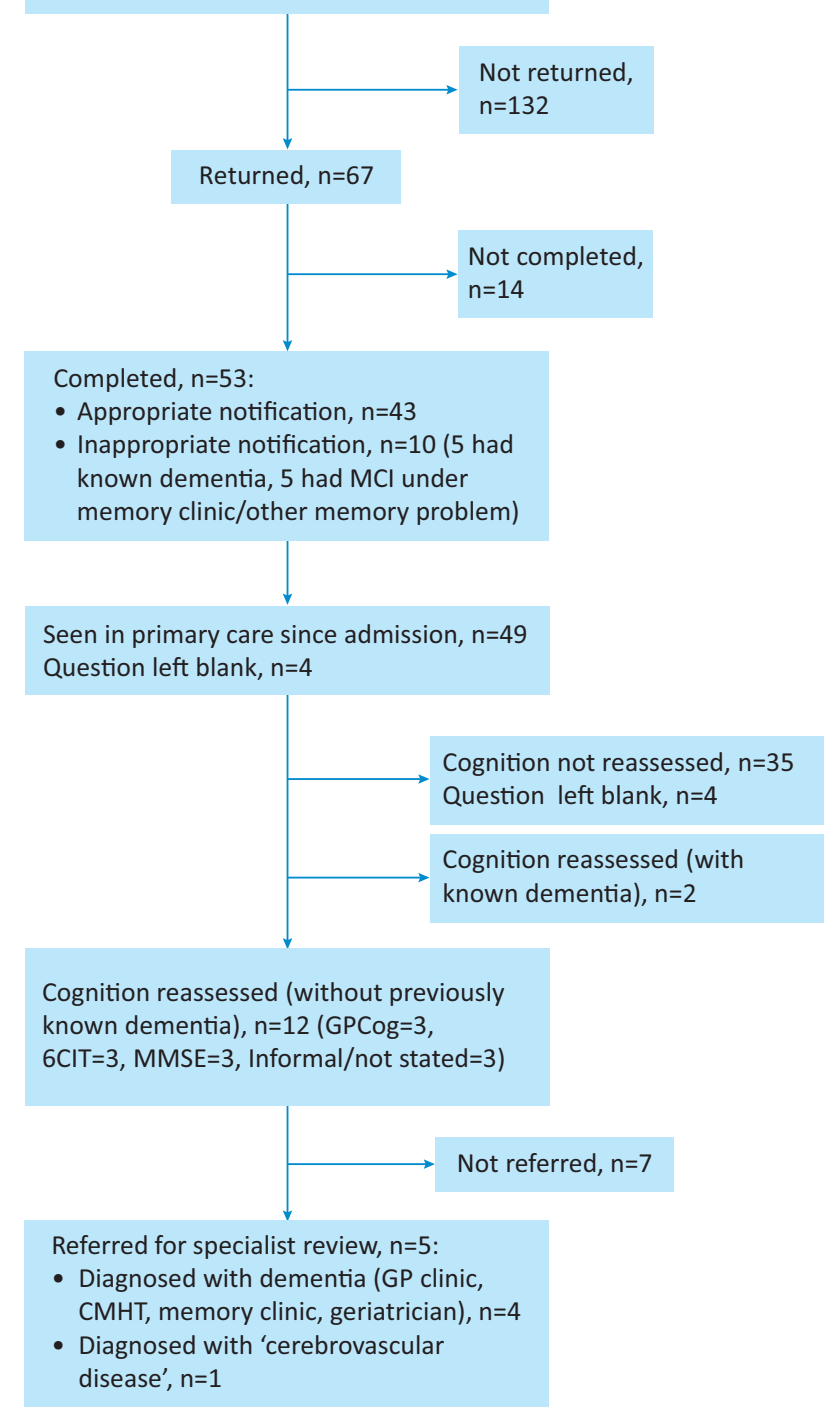

Fig 2. Flow diagram of questionnaire data. $6 \mathrm{CIT}=$ six-item cognitive impairment test; $\mathrm{CMHT}=$ community mental health team; $\mathrm{GPCog}=\mathrm{GP}$ cognitive screen; $\mathrm{MCI}$ = mild cognitive impairment; $\mathrm{MMSE}$ = mini-mental state examination.

Free text comments extracted from questionnaires showed some clear themes (Box 1). Reasons for lack of cognitive reassessment/specialist referral were given in 25 patients. In 15 cases, it was felt to be inappropriate as the patient was too unwell or frail, other pathologies were the major clinical issue or the memory problem was not the principal concern. Some patients/families declined specialist referral or the patient was not worried sufficiently about their memory to want reassessment/referral $(n=5)$. Some GPs $(n=5)$ expected cognitive impairment in older patients in the context of acute illness and felt that only scores obtained during the medically stable state would be helpful. Process issues highlighted included poor 


\section{Box 1. Comments from GPs, reproduced verbatim and grouped by theme, including reasons for lack of} cognitive reassessment/specialist referral

\section{Clinically inappropriate}

$>$ End-stage renal failure, not for resus or any further hospital contact - terminal care.

$>$ Having treatment for oesophageal Ca so not considered appropriate.

> Seen frequently by GP who has known pt for years. Further decline from CVA and death in July - focus not on long-term dementia care but short-term supportive measures the priority.

$>$ Extreme frailty. Already had her care needs met in nursing home.

$>$ Patient well, no obvious cognitive issue on GPCog.

$>$ Patient had returned to pre-admission levels of cognition.

> Memory problems/confusion have not been an apparent problem. There have been several other ongoing and new physical problems, which have dominated his consultations.

$>$ Too frail and ill - died.

> Patient was on palliative framework at the time of admission for treating a reversible problem. Treatment was symptomatic/palliative.

> This patient has known encephalopathy causing confusion, low AMTS.

$>$ Has had too many other concerns, patient does not feel concerned enough at present.

> Age 98, resident in a care home not for resus, ie of no benefit.

$>$ Advanced age, multiple pathologies.

> Advanced age, severe COPD, wellbeing not affected.

$>$ Not felt to be significant on holistic approach.

\section{GP's expected abnormal cognition in context of acute illness}

$>$ Patient had sepsis due to renal abscess at time of admission.

$>\mathrm{V}$ frail. Dip in cognition attributed to acute admission with AKI.

> Patient admitted with septicaemia leading to his poor cognition.

> An admission AMTS is unhelpful. Many elderly patients are confused when acutely unwell. A discharge AMTS would be much more useful and we might then follow up.

$>$ Unwell at time, no concern since.

\section{Patient/family decision}

> Patient does not feel concerned enough at present. Further admission with GI bleeds.

$>$ Patient has refused three times for memory referral, including home visit assessment by specialist team. Son aware.

$>$ Patient declined any further intervention when assessed.

$>$ Did not express concerns with memory at consultation.

> Daughter declined referral to memory clinic because of mother's frailty.

\section{Process issues}

> Patients should be instructed to make an appointment to see their GP a few weeks following discharge to discuss this.

> I do not have spare time in GP to review the patient records of five patients in detail. If you would like info that is accurate you will need to send some admin staff into surgeries to do this work.

$>$ Discharge letter v poor, diagnosis confusion, no other details. No AMTS score sent to GP.

> If the person doing the screening had checked with the next of kin first, or with us, they would not have duplicated the process! This needs to be done in a more joined up manner*

\section{GP notes re ongoing management}

> There is no letter alerting to her low AMTS score. I will follow up dementia screening on next encounter with patient - note made on her record to do this.

$>$ GP to assess at next review, (seen by DN). Patient to be reviewed as planned (no concern re cognition by DN).

*This was a patient who had received a dementia diagnosis in memory clinic just prior to admission.

AKI = acute kidney injury; AMTS = Abbreviated Mental Test Score; $\mathrm{Ca}=$ cancer; $\mathrm{COPD}=$ chronic obstructive pulmonary disease; $\mathrm{CVA}=$ cerebrovascular accident; $\mathrm{DN}=$ district nurse; GI = gastrointestinal; GPCog = GP cognitive screen; $p t=$ patient; $v$ = very 
communication between secondary and primary care and insufficient resources to enable questionnaire completion.

\section{Discussion}

Our study showed that the majority of older patients identified in hospital as at risk of dementia had been reviewed by primary care in the 6 months following discharge and although only around a quarter had had a cognitive reassessment, nonreassessment/referral was often for a valid clinical reason.

The effective transmission of information on cognitive status over the primary/secondary care interface should be an integral part of individualised older patient care. However, our findings revealed that this was often suboptimal as is seen in other conditions. ${ }^{20}$ Just under a fifth of patients already had a cognitive diagnosis (either dementia or a milder memory problem) that had been unknown to the hospital team. Lack of access to electronic records across different healthcare providers likely contributed to this issue but the study design did not allow us to examine this further. Similarly, in a small number of cases, primary care practices had not received or could not find the notification letter containing the cognitive screening results. Where the discharge information was received, letters were usually incorporated into the primary care patient record/ documents file but this was not usually done in a way that enabled easy searching or flagging of those at-risk since only $<5 \%$ were coded.

Reasons given by GPs for lack of cognitive reassessment or specialist referral indicated that in many cases it was felt to be clinically inappropriate because the patient was nearing the end of life or because referral was declined by patients or carers. However, some GPs appeared unaware of the prognostic implications for risk of ongoing/future cognitive decline and for recurrent episodes. ${ }^{5,714,21,22}$ The relationship between delirium/ transient cognitive impairment and dementia is analogous to that between acute kidney injury and chronic kidney disease - acute kidney injury is considered a risk marker for the development of chronic kidney disease and for chronic kidney disease progression. ${ }^{23-25}$ Current guidelines require that acute kidney injury episodes are communicated to GPs on discharge to target monitoring of renal function and enhance awareness of vulnerable patients ${ }^{25}$ although this may not always be done reliably. ${ }^{20}$ In contrast, neither delirium nor transient cognitive impairment have been incorporated into primary care systems designed to flag those at risk of dementia ${ }^{26}$ and existing risk scores for dementia are focused on vascular risk factors, which may not be as relevant in older versus younger patients. ${ }^{27-29}$

There are some limitations to our study. The sample size was reduced since only a minority of primary care practices returned the questionnaires and some questionnaires were returned blank. However, there was no difference in age and AMTS score in those with/without a returned questionnaire. Second, questionnaire return may have been more likely from GPs interested in cognitive disorders resulting in possible bias in reported reassessment rates. Third, no reason for lack of reassessment/referral was given in many cases, resulting in a possible lack of capture of other reasons for non-reassessment. Study strengths include the use of questionnaires to obtain quantitative and qualitative information not routinely available through electronic records or routine administrative coding.

\section{Conclusions}

GPs are well placed to decide with patients and families whether specialist memory referral is desirable in those identified as at risk and routine referral during acute hospital admission would appear unjustified. However, the prognostic value of worsened cognition in the context of acute illness appears underrecognised by some. In-hospital identification of at-risk status could be listed/coded in the primary care record to highlight vulnerability to progressive cognitive decline and to facilitate delirium prevention/recognition and end-of-life care planning.

\section{Conflicts of interest}

The authors have no conflicts of interest to declare.

\section{Author contributions}

AP and BK collected data, RW analysed data, and STP conceived and designed the audit and wrote the manuscript.

\section{Acknowledgements}

STP is supported by the NIHR Oxford Biomedical Research Centre. There was no specific funding for this study and the sponsors had no role in the study design or analyses.

\section{References}

1 Sampson EL, Blanchard MR, Jones L et al. Dementia in the acute hospital: prospective cohort study of prevalence and mortality. $\mathrm{Br} \mathrm{J}$ Psychiatry 2009;195:61-6.

2 Future Hospital Commission. Future hospital: caring for medical patients. A report from the Future Hospital Commission to the Royal College of Physicians. London: RCP, 2013.

3 Pendlebury ST, Klaus SP, Mather M, de Brito M, Wharton R. Routine cognitive screening in older patients admitted to acute medicine: Abbreviated Mental Test Score (AMTS) and subjective memory complaint versus Montreal Cognitive Assessment and IQCODE. Age Ageing 2015;44:1000-5.

4 Jackson TA, MacLullich AM, Gladman JR, Lord JM, Sheehan B. Undiagnosed long-term cognitive impairment in acutely hospitalised older medical patients with delirium: a prospective cohort study. Age Ageing 2016;45:493-9.

5 Inouye SK, Westendorp RG, Saczynski JS. Delirium in elderly people. Lancet 2014;383:911-22.

6 Pendlebury ST, Lovett NG, Smith SC et al. Observational, longitudinal study of delirium in consecutive unselected acute medical admissions: age-specific rates and associated factors, mortality and re-admission. BMJ Open 2015;5:e007808.

7 Pendlebury ST, Wadling S, Silver LE, Mehta Z, Rothwell PM. Transient cognitive impairment in TIA and minor stroke. Stroke 2011;42:3116-21.

8 NHS Commissioning Board. Commissioning for quality and innovation (CQUIN): 2013/14 guidance. Draft - December 2012. Leeds: NHS England, 2012. www.england.nhs.uk/wp-content/uploads/2012/12/ cquin-guidance.pdf [Accessed 17 August 2017].

9 Brunet MD, McCartney M, Heath I et al. There is no evidence base for proposed dementia screening. BMJ 2012;345:e8588.

10 Brunet M. Targets for dementia diagnoses will lead to overdiagnosis. BMJ 2014;348:g2224.

11 Aji BM, Larner AJ. Screening for dementia: is one simple question the answer? Clin Med 2015;15:111-2. 
12 NHS England. Dementia Assessment and Referral 2016-17. www.england.nhs.uk/statistics/statistical-work-areas/dementia/ dementia-assessment-and-referral-2016-17/ [Accessed 19 July 2017].

13 National Institute for Health and Care Excellence. Delirium, diagnosis, prevention and management. NICE clinical guideline No 103. London: NICE, 2010.

14 Rockwood K, Cosway S, Carver D et al. The risk of dementia and death after delirium. Age Ageing 1999;28:551-6.

15 Eeles EM, White SV, O'Mahony SM, Bayer AJ, Hubbard RE. The impact of frailty and delirium on mortality in older inpatients. Age Ageing 2012;41:412-6.

16 Pendlebury ST, Klaus SP, Thomson RJ et al. Methodological factors in determining risk of dementia after transient ischemic attack and stroke: (iii) applicability of cognitive tests. Stroke 2015;46:3067-73.

17 Pendlebury ST, Lovett NG, Smith SC, Wharton R, Rothwell PM. Delirium risk tratification in consecutive unselected admissions to acute medicine: validation of a susceptibility score based on factors identified externally in pooled data for use at entry to the acute care pathway. Age Ageing 2017;46:226-31

18 Hodkinson HM. Evaluation of a mental test score for assessment of mental impairment in the elderly. Age Ageing 1972;1:233-8.

19 Inouye SK, van Dyck CH, Alessi CA et al. Clarifying confusion: the confusion assessment method. A new method for detection of delirium. Ann Intern Med 1990;113:941-8.

20 Greer RC, Liu Y, Crews DC et al. Hospital discharge communications during care transitions for patients with acute kidney injury: a cross-sectional study. BMC Health Serv Res 2016;16:449.

21 Fong TG, Jones RN, Shi P et al. Delirium accelerates cognitive decline in Alzheimer disease. Neurology 2009;72:1570-5.
22 van Gool WA, van de Beek D, Eikelenboom P. Systemic infection and delirium: when cytokines and acetylcholine collide. Lancet 2010;375:773-5.

23 Hsu RK, Hsu CY. The role of acute kidney injury in chronic kidney disease. Semin Nephrol 2016;36:283-92.

24 Sawhney S, Mitchell M, Marks A et al. Long-term prognosis after acute kidney injury (AKI): what is the role of baseline kidney function and recovery? A systematic review. BMJ Open 2015;4:e006497.

25 Sawhney S, Marks A, Black C. Discharge after acute kidney injury recognising and managing risk. Clinical Focus Primary Care 2016;9:124-33.

26 NHS England. Enhanced Service Specification: facilitating timely diagnosis and support for people with dementia 2015/16. Leeds: NHS England, 2015.

27 Stephan BC, Kurth T, Matthews FE, Brayne C, Dufouil C. Dementia risk prediction in the population: are screening models accurate? Nat Rev Neurol 2010;6:318-26.

28 Barnes DE, Beiser AS, Lee A et al. Development and validation of a brief dementia screening indicator for primary care. Alzheimers Dement 2014;10:656-665.e1.

29 Walters K, Hardoon S, Petersen I et al. Predicting dementia risk in primary care: development and validation of the Dementia Risk Score using routinely collected data. BMC Med 2016;14:6.

Address for correspondence: Dr Sarah Pendlebury, Centre for Prevention of Stroke and Dementia, Level 6 West Wing, John Radcliffe Hospital, Oxford OX3 9DU, UK. Email: sarah.pendlebury@ndcn.ox.ac.uk 